Revista de Investigación Universitaria, 2015, Vol. 4 (2): 79-82

ISSN: 2312-4253 (Versión impresa) ISSN: 2078-4015 (Versión digital)

\title{
Evaluación de la calidad de aire por partículas menores a 10 microgramos y nivel de riesgo basado en el índice de calidad de aire en la Universidad Peruana Unión - FT \\ Evaluation of the air quality by particles smaller than $\mathbf{1 0}$ micrograms and level of risk based on the air quality index in Universidad Peruana Unión - FT
}

\author{
Pérez Carpio, Jackson Edgardo; Cárdenas Soto, Rolando y Almestar Villegas, Carmelino \\ EP. Ingeniería Ambiental, Facultad de Ingeniería y Arquitectura, Universidad Peruana Unión \\ Recibido 10 de octubre del 2015-Aceptado 4 de diciembre del 2015
}

\begin{abstract}
Resumen
El objetivo de la investigación es evaluar la concentración de partículas menores a 10 microgramos y comparar con el índice de calidad del aire de la agencia de protección ambiental, el tipo de investigación no experimental, descriptivo comparativo de corte trasversal, porque está dirigido a responder por las causas de los eventos y fenómenos físicos y solo se determina el grado de relación de las variables de estudio: calidad de aire por partículas menores de 10 microgramos y nivel de riesgo basado en el índice de calidad de aire, se han realizado el monitoreo teniendo en cuenta el protocolo de calidad de aire, de acuerdo a los resultados obtenidos valores de PM10 se encuentran por debajo de los ECA, interpretándose como una calidad aceptable; y, según los ICA, corresponde a una calidad de aire que no representa un riesgo para la salud.
\end{abstract}

Palabras clave: Particulas, calidad de aire, estándar de calidad de aire, índice de calidad de aire.

\begin{abstract}
The aim of the investigation is to evaluate the concentration of particles smaller than 10 micrograms and to compare with the air quality index of the environmental protection agency, the type of non-experimental, comparative cross-sectional descriptive research, because it is aimed at responding By the causes of the events and physical phenomena and only determines the degree of relation of the study variables: air quality by particles smaller than 10 micrograms and level of risk based on the air quality index, the monitoring Taking into account the air quality protocol, according to the results obtained PM10 values are below the RCTs interpreted as an acceptable quality; And according to the ICA corresponds to a quality of air that does not represent a health risk.
\end{abstract}

Keywords: Particles, air quality, standard of air quality, air quality index.

\section{Introducción}

El campus de la Universidad Peruana Unión, Filial Tarapoto, cuenta con alumnos y personal que viven y laboran en la institución, tiene una población aproximadamente de 1500 personas entre estudiantes y personal se realizan diferentes actividades como académicas, espirituales y físicas, este trabajo de investigación ayudará a dar a conocer cuál es el grado de contaminación de la calidad de aire por material particulado, menores a 10 microgramos.

La resolución Nº 339-2012, emitido por el Ministerio del Ambiente Perú (2012), la ciudad de Tarapoto ha sido considerada dentro de los dieciocho (18) nuevas zonas de atención prioritaria en las cuales se evaluará y formulará los planes de acción para el mejoramiento de la calidad del aire. Para esto se deberá tomar

Correspondencia al autor: email: jacksonepcl@gmail.com 
Pérez, J.; Cárdenas, R. y Almestar, C.

como referencia el incremento de la actividad industrial y comercial que se realiza en ellas el tamaño del parque automotor y la población vulnerable.

En la Resolución Ministerial N 307 - 2015 del Ministerio del Ambiente Perú (2015) artículo 1², menciona que se debe aprobar el plan de acción para la mejora de la calidad del aire en la zona de atención prioritaria de la cuenca atmosférica San Martín. De la misma manera, menciona que en el departamento de San Martín se ha incrementado la cantidad de vehículos motorizados, por cada mil habitantes. Ha tenido una tendencia de crecimiento de 12.94 en el año 2009 a 15.55 vehículos por cada 1000 habitantes en el año 2012. El parque automotor de la zona de atención priorizada, al año 2012, fue de 27000 vehículos de transporte terrestre, de los cuales 5885 corresponden a las categorías $\mathrm{M}$ y N (autos, station wagon, camionetas, micro-bus, camión y semi-trayler), de acuerdo al Reglamento Nacional de Vehículos (Decreto Supremo N 058-2003-MTC); y 23000 vehículos pertenecen a la Categoría L (mototaxis y motos lineales), cifra bastante alta, debido a que en el caso de Tarapoto, Morales y La Banda de Shilcayo, por sus características geográficas el transporte público y privado se realiza en forma masiva en vehículos menores (motos lineales y mototaxis o motocar.
Según el Decreto Supremo Nº 003 - 2008, los estándares de calidad de aire (ECA), elaborado por el Ministerio del Ambiente Perú (2008), se refieren a valores que no representen riesgo significativo para la salud de las personas ni para el ambiente, siendo que el concepto de valor guía de la calidad del aire, desarrollado por la Organización Mundial de la Salud (OMS), se refiere al valor de la concentración de los contaminantes en el aire por debajo del cual la exposición no representa un riesgo significativo para la salud.

Airnow (2014) menciona que el AQI es un índice para la presentación de informes de calidad de aire te dice cuan limpio o contaminado es su aire, y qué efectos en la salud asociados podrían ser una preocupación para usted. El AQI se enfoca en los efectos sobre la salud que pueden surgir dentro de unas pocas horas o días, después de respirar aire contaminado. La EPA calcula el AQI por cinco principales contaminantes del aire, regulados por la Ley de Aire Limpio: ozono a nivel del suelo, la contaminación de partículas (también conocida como material particulado), monóxido de carbono, dióxido de azufre y dióxido de nitrógeno. Para cada uno de estos contaminantes, la EPA ha establecido estándares nacionales de calidad del aire para proteger la salud pública.

Tabla 1

Índice de Calidad de Aire para PM10 basado en la EPA.

\begin{tabular}{llll}
\hline Índice de calidad & Nivel de riesgo & \multicolumn{2}{c}{ Acciones de control de la contaminación } \\
\cline { 2 - 3 } & Calificación & Escala de color & \\
\hline $0-50$ & Buena & Verde & $\begin{array}{l}\text { La calidad del aire es satisfactoria. } \\
\text { La calidad del aire es aceptable, puede causar efectos } \\
\text { moderado en la salud de personas sensibles. }\end{array}$ \\
$101-200$ & Regular & Amarillo & $\begin{array}{l}\text { Miembros de grupos sensibles pueden experimentar } \\
\text { efectos sobre la salud. }\end{array}$ \\
$201-300$ & Mala & Naranja & $\begin{array}{l}\text { Alerta de salud, efectos sobre salud cardiovascular y asma. } \\
\text { Advertencia de condición de emergencia, salud de la } \\
\text { población en riesgo. }\end{array}$ \\
\hline
\end{tabular}

Fuente: Environmental Protection Agency

El Ministerio del Ambiente Perú (2015), según la R.M 307 - 2015 MINAN, en el Plan de acción para la mejora de la calidad del aire en la zona de atención prioritaria de la cuenca atmosférica de San Martín en el 2013 se realizó el monitoreo de calidad de aire de PM10, tal como se muestra en la tabla 2. 
Evaluación de la calidad de aire por partículas menores a 10 microgramos y nivel de riesgo basado en el índice de calidad de aire en la Universidad Peruana Unión - FT

Tabla 2

Resultados de Monitoreo de Calidad de Aire- ZAP San Martín (2013)

\begin{tabular}{|c|c|c|c|c|c|}
\hline \multicolumn{6}{|c|}{ Resultados Monitoreo de Calidad de Aire } \\
\hline Estación de monitoreo & Fecha de monitoreo & $\mathbf{P M}_{10}(\mathbf{u}$ & $\begin{array}{l}\text { Dióxido de } \\
\text { nitrógeno }\end{array}$ & $\begin{array}{l}\text { Dióxido de } \\
\text { azufre }\end{array}$ & $\mathbf{P M}_{2.5}$ (ug/m3) \\
\hline \multirow{3}{*}{$\begin{array}{l}\text { E3 Municipalidad } \\
\text { Distrital de Morales }\end{array}$} & $18-19 / 11 / 13$ & 30.88 & 23.31 & 4.29 & 12.91 \\
\hline & $19-20 / 11 / 13$ & 27.30 & 47.38 & --- & --- \\
\hline & $20-21 / 11 / 13$ & 36.11 & 24.82 & 6.46 & 16.24 \\
\hline ECA para Aire & & $150(1)$ & 200(1) & $\begin{array}{l}20(2) \\
80\end{array}$ & $\begin{array}{l}25(2) \\
50\end{array}$ \\
\hline
\end{tabular}

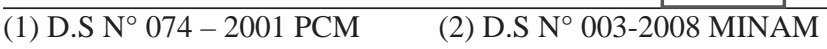

Fuente: MINAM, Dirección General de Calidad Ambiental

No existen, en la actualidad, estudios realizados de monitoreo de calidad de aire en distrito de Morales y en campus de la Universidad, esté trabajo de investigación ayudará a conocer cuál es el índice de calidad de aire y a qué nivel de riesgo se encuentra expuesto la población estudiantil y el personal.

\section{Método}

\section{Tipo de investigación}

La investigación es de tipo cuantitativa no experimental, porque se observan los fenómenos tal como se dan en su contexto natural, para posteriormente analizarlos. Según Hernandez Fernández y Baptista (2010, p. 4).

\section{Diseño de investigación}

El presente estudio tiene un enfoque de tipo no experimental, descriptivo comparativo de corte transeccional o trasversal; es de tipo no experimental porque es aquella que se realiza sin manipular deliberadamente variables. Es decir, la investigación donde no se hace variar intencionalmente las variables independientes, se observa fenómenos tal como se dan en su contexto natural para después analizarlos, el diseño es transversal porque recolectan datos en un solo momento, en un tiempo único, su propósito es describir variables y analizar su incidencia e interrelación en un momento dado. En cierta medida los diseños trasversales tienen como objetivo indagar la incidencia y los valores en que se manifiesta una o más variables, se puede decir que esta investigación también es correlacional, puesto que estos diseños describen relacio- nes entre dos o más categorías, conceptos o variables en un momento determinado (Hernández, Fernández y Baptista, 2010).

\section{Metodología de Monitoreo}

- Conocer las medidas de seguridad.

- Abrir la carcasa del equipo y fijarla en la parte de atrás.

- Quitar los cuatro tornillos de sujeción del portafiltro.

- Colocar el filtro registrando su peso inicial.

- Ajustar el portafiltro apretando firmemente los tornillos

- Cerrar la carcasa del equipo

- Programar el tiempo para un período de 24 horas o anotar la lectura inicial del tiempo

- Prender el equipo.

- Tomar la lectura de la presión inicial.

- Una vez finalizado el tiempo retirar el filtro.

\section{Metodología de análisis}

- Codificar el filtro con mucho cuidado para que este no se contamine o no se rompa. Se recomienda confeccionar un portafiltro (no tocar o manipular directamente los filtros).

- Colocar el filtro en la estufa por un periodo de 24 horas a $30^{\circ} \mathrm{C}$, el filtro se pesa y se almacena durante 24 horas a temperaturas antes indicadas, para eliminar la humedad adquirida por efectos del ambiente.

- Se pesa nuevamente el filtro patrón o de referencia, el objetivo es balancear la ganancia o pérdida de la humedad ambiental durante el período de muestreo. 
- Después de haber llenado la hoja de muestreo, se utiliza la caja Petri para trasladar el filtro al sitio de muestreo.

- Se anota el tiempo final de la medición de 24 horas.

- El filtro expuesto se retira con una pinza colocándolo en una caja petri y se etiqueta.

- Se coloca el filtro en la estufa por 24 horas a 30 ${ }^{\circ} \mathrm{C}$ para eliminar la humedad, después se coloca en un desecador por 30 minutos.

- El filtro se pesa tres veces del valor promedio, se resta luego el peso del valor promedio

\section{Selección del punto de muestreo}

Se han determinado tres puntos de monitoreo dentro del campus de la Universidad Peruana Unión y los monitoreos se han realizado (tabla 3 ).

Tabla 3

Ubicación de Puntos de Monitoreo

\begin{tabular}{clrl}
\hline \multirow{2}{*}{$\begin{array}{c}\text { Estación de } \\
\text { monitoreo }\end{array}$} & \multicolumn{2}{c}{ Coordenadas } & Referencia \\
\cline { 2 - 3 } & $\mathbf{N}$ & \multicolumn{1}{c}{$\mathbf{S}$} & \\
\hline P1 & 0345546 & 9284387 & Costado del pabellón A \\
P2 & 03645634 & 9284332 & Costado del pabellón B \\
P3 & 0345621 & 9284419 & $\begin{array}{c}\text { Costado del Laboratorio } \\
\text { de Ing Ambiental }\end{array}$ \\
\hline
\end{tabular}

\section{Resultados y Discusión}

\section{Resultado de monitoreo de calidad de aire}

Se han realizado los monitoreos de calidad de aire de material particulado de 10 microgramos en el campus de la Universidad Peruana Unión FT, en tres puntos de monitoreos y se han obtenidos resultados menores a la normativa de comparación de calidad de aire D.S $\mathrm{N}^{\circ}$ 074-2001, tal como se muestra en la tabla 4, según los ICA corresponde a una calidad de aire que no representa un riesgo para la salud.

Tabla 4

Resultado de la concentración calculada

\begin{tabular}{|c|c|c|c|c|c|}
\hline \multirow{2}{*}{ Punto de muestra } & \multicolumn{2}{|l|}{ Peso } & \multirow{2}{*}{$\begin{array}{l}\text { Concen- } \\
\text { tración }\end{array}$} & \multirow{2}{*}{ ECA } & \multirow{2}{*}{$\begin{array}{l}\text { Nivel de } \\
\text { Riesgo } \\
\text { Califi- } \\
\text { cación }\end{array}$} \\
\hline & Inicial & Final & & & \\
\hline Costado del pabellón A & $4.4714 \mathrm{~g}$ & $4.5081 \mathrm{~g}$ & $15.8 \mu \mathrm{g} / \mathrm{m}^{3}$ & \multirow{3}{*}{$150 \mu \mathrm{g} / \mathrm{m}^{3}$} & \multirow{3}{*}{ Buena } \\
\hline Costado del pabellón B & $4.3856 \mathrm{~g}$ & $4.4785 \mathrm{~g}$ & $40.1 \mu \mathrm{g} / \mathrm{m}^{3}$ & & \\
\hline $\begin{array}{l}\text { Costado del } \\
\text { Laboratorio de Ing. } \\
\text { Ambiental }\end{array}$ & $4.4416 \mathrm{~g}$ & $4.4921 \mathrm{~g}$ & 21.8. $\mu \mathrm{g} / \mathrm{m}^{3}$ & & \\
\hline
\end{tabular}

\section{Conclusiones}

Se han comparado los resultados obtenidos de los contaminantes de partículas menores a 10 microgramos con el índice de calidad de aire.

De acuerdo a los resultados obtenidos en los 3 puntos de monitoreo, realizado en el campus de la Universidad Peruana Unión Filial Tarapoto, no excedieron el nivel de referencia establecido por el D.S N ${ }^{\circ} 074-$ 2001, ya que las concentraciones evaluados se encuentran por debajo del estándar de calidad de aire $150 \mu \mathrm{g} / \mathrm{m}^{3}$

Comparando con los valores del índice de calidad de aire de la agencia y protección ambiental, la calificación del nivel de riesgo es buena y se puede afirmar que la calidad de aire es satisfactoria.

\section{Recomendaciones}

Continuar realizando los monitoreos de calidad de aire en el campus de la Universidad, para así poder tomar medidas correctivas si amerita el caso. Realizar monitoreos de gases de combustión.

\section{Referencias}

Airnow. (2014). Air Quality Index (AQI) - A guide to air quality and your health. Recuperado de (http://www. airnow.gov/index.cfm?action=aqibasics.aqi\#mod).

Hernandez, Roberto, Carlos Fernández, and Pilar Baptista. (2010). Metodología de la investigación. Quinta edi. edited by Jesús Mares Chacón. Mexico.

Ministerio del Ambiente Perú. (2008). “Estándares de calidad ambiental para aire Decreto Supremo No 003-2008-MINAM.” 1-3. Recuperado de (http://www. senace.gob.pe/normativa/normas-ambientales-transversales/estandares-de-calidad-ambiental/).

Ministerio del Ambiente Perú. (2012). Resolución Ministerial No 3392012 MINAN. Recuperado de (http:// www.minam.gob.pe/disposiciones/resolucion-ministerial-n-339-2012-minam/).

Ministerio del Ambiente Perú. (2015). Resolución Ministerial No307 2015 MINAN. Recuperado de (http:// www.minam.gob.pe/wp-content/uploads/2015/11/ RM-307-2015-MINAM.pdf).

Ministerio del Ambiente Perú. (2012). Resolución Ministerial No339 2012 MINAN. Perú. Recuperado de http:// www.minam.gob.pe/disposiciones/resolucion-ministerial-n-339-2012-minam/ 